\title{
Confronting and Coping with Multidrug-Resistant Tuberculosis: Life Experiences in Thailand
}

Qualitative Health Research

2021, Vol. 0(0) I-9

(C) The Author(s) 2021

\section{(c) (i) (5)}

Article reuse guidelines:

sagepub.com/journals-permissions

DOI: I0.1 I77/I04973232। I049777

journals.sagepub.com/home/qhr

(S)SAGE

\author{
Samorn Numpong' $\odot$, Mondha Kengganpanich², Jaranit Kaewkungwal', \\ Wirichada Pan-ngum ${ }^{\oplus}{ }^{\odot}$, Udomsak Silachamroon ${ }^{3}$, Yuthichai Kasetjaroen ${ }^{4}$, and \\ Saranath Lawpoolsri'
}

\begin{abstract}
In this article, we aimed to understand the life experiences of Thai persons diagnosed with multi-drug-resistant tuberculosis (MDR-TB). A qualitative study using a face-to-face in-depth interview was conducted at a hospital in Thailand which has the highest prevalence of MDR-TB in the country between January and February 2019. Twenty persons living with MDR-TB in Thailand were purposively selected to represent a variety of experiences based on different gender, ages, and treatment phases. Qualitative data were transcribed and thematic analysis was applied to identify common themes and sub-themes. The results indicated that all participants faced emotional difficulties, such as fear of death, fear of stigmatization, confusion, and sadness when first knowing of their diagnosis. Family and social support were the main ways that the patients coped with difficult situations. Suicidal ideas were more prevalent among patients with poor family support. Screening for mental health problems should be routinely performed in MDR-TB patients. Proper health education should be provided to patients and families to reduce emotional difficulties and stigmatization.
\end{abstract}

\section{Keywords}

life experience, self-management, tuberculosis patients, multi-drug-resistant tuberculosis, Thailand

\section{Introduction}

People diagnosed with multi-drug-resistant tuberculosis (MDR-TB) require long-term treatment of about 1824 months with a variety of medications. These patients are prone to mental health problems, such as anxiety and depression. Side effects of the complex treatments can cause adverse mental health reactions. For example, injectable TB drugs may cause severe side effects, such as hearing loss and optic neuritis (Bureau of Tuberculosis, 2015). Depression has been reported as a side effect of antiTB drugs, especially cycloserine (Borisov et al., 2019). Additionally, $2.4 \%$ of patients with MDR-TB and human immunodeficiency virus (HIV) co-infection who received anti-retroviral drugs, such as efavirenz, have experienced severe depression (World Health Organization, 2014). In addition, persons living with MDR-TB have reported stigma, discrimination, and fear of disease contagiousness, which may lead to self-isolation from family and friends (Alene et al., 2018; Morris et al., 2013; Thomas et al., 2016). A study in Peru reported that MDR-TB patients developed depression, anxiety, and psychosis during the treatment period about $13.3 \%, 12 \%$, and $12 \%$, respectively, (Vega et al., 2004). Social and economic factors were also associated with the development of anxiety in MDR-TB patients because approximately $70 \%$ of MDR-TB patients lost their job after being diagnosed (Walker et al., 2018). Mental health illnesses influenced medication non-

\footnotetext{
'Faculty of Tropical Medicine, Department of Tropical Hygiene, Mahidol University, Bangkok, Thailand

${ }^{2}$ Faculty of Public Health, Department of Health Education and Behavioral Sciences, Mahidol University, Bangkok, Thailand ${ }^{3}$ Faculty of Tropical Medicine, Department of Clinical Tropical Medicine, Mahidol University, Bangkok, Thailand

${ }^{4}$ AIDS, Tuberculosis and Sexually Transmitted Disease Control Division, The Health Department, Bangkok Metropolitan Administration, Bangkok, Thailand

Corresponding Author:

Saranath Lawpoolsri, Faculty of Tropical Medicine, Department of Tropical Hygiene, Mahidol University, 420/6 Ratchathewi, Bangkok 10400, Thailand.

E-mail: saranath.law@mahidol.ac.th
} 
adherence. Consequently, treatment non-adherence led to poor treatment outcomes (Grenard et al., 2011).

Although the World Health Organization has recommended screening anxiety and depression among MDR-TB patients, mental health problems among MDR-TB patients are not well recognized in many countries, including Thailand. Case management guidelines of MDR-TB mostly focused on medication treatment and response. The diagnosis and treatment of tuberculosis are covered by the national insurance schemes in Thailand. Because of the high workload of health care providers, the patients usually do not have an opportunity to discuss their worries and life change during the treatment with doctors and nurses at TB clinics.Moreover, a routine anxiety and depression screening was not included in the national MDR-TB treatment guideline. The research question remained whether how people diagnosed with MDR-TB confronted and managed their difficult situation. Therefore, the objectives of this study were to understand the life experiences of Thai persons' diagnosis with MDR-TB and how they managed their mental health problems, particularly anxiety and depression. The findings from this study may provide insights into the hidden mental health problems among this high-risk population and highlight the importance of including mental healthcare in the MDR-TB case management guideline, with the goal of improving the quality of life and treatment outcomes of MDR-TB patients.

\section{Method}

\section{Study Design}

This qualitative descriptive study involved in-depth interviews of people diagnosed with MDR-TB in a hospital in Thailand, which has the highest prevalence of MDRTB in the country (Jiraphongsa et al., 2011; Kittyanun, 2007; Thanprasertsuk et al., 2017). Life experience of persons living with MDR-TB was comprehensively summarized, without pre-specified theoretical commitment (Lambert \& Lambert, 2012). The in-depth interviews were conducted from January to February 2019.

\section{Study Population and Recruitment}

The study hospital has about 200 newly registered TB patients annually, with about 35-40 newly diagnosed MDRTB cases registered each year, according to the hospital's report in 2017. Generally, the TB clinic is operated on alternate weeks. Patients with MDR-TB are required to visit the clinic every 2 weeks during the intensive treatment phase. During the maintenance phase, they are required to come to the clinic once per month. The clinic currently follows 37 persons with MDR-TB. Approximately 20-30 patients are seen during the weekly TB clinics. Participants were selected using purposive sampling method. The TB clinic nurse listed patients to be representative of people diagnosed with MDR-TB by including all sex, age groups, both intensive and maintenance treatment phases, and levels of treatment compliance. This method led to a selection of a mixed sample in terms of varied demographic (age and gender), treatment phases, and reported mental health issues. The inclusion criteria included MDR-TB patients with Thai nationality, aged $\geq 18$ years, and willing to participate in the study. Prisoners and those who were unable to provide the information were excluded. The sample size was determined by data saturation and representativeness, and finally, a total of 20 MDR-TB patients participated in the in-depth interviews.

\section{Data Collection}

All participants were asked to complete the selfassessment using the Thai version of the Hospital Anxiety and Depression Scale (HADS) before an in-depth interview; 14 items were separated into two parts: odd numbers of items for anxiety and even numbers for depression assessment (Nilchaikovit et al., 1996). An HADS score of $\leq 7$ is defined as no anxiety or depression. A patient with a score of $8-10$ is defined as doubtful case which requires further confirmation by a psychiatrist or a psychologist. And a score of $\geq 11$ suggests that the participant is likely to have anxiety or depression (Zigmond \& Snaith, 1983). Six patients who were illiterate had an interviewer read the HADS questions to them. An interview guide with open-ended questions was developed for the in-depth interviews. It consisted of three major questions and various minor questions about the patient's life experience in confronting MDR-TB, anxiety and depression issues, how to manage their emotions, and demographic characteristics. Experts in tuberculosis treatment and qualitative research were invited to review the interview guide. Trained interviewers, who were not the nurses or providers at TB clinic, administered the in-depth face-to-face interviews. Treatment information and adverse drug reactions were obtained from the patients' medical records. The interviews were conducted in Thai language and took approximately $30-40$ minutes per patient. The interview was conducted in a private area in the infectious control section of the hospital. Informed consent was obtained before the interview. All interviews were recorded with permission for further transcription and data extraction.

\section{Data Analysis}

Thematic analysis was performed to explore the patterns of the qualitative data. A full transcription of the audio recording of the interview was conducted by the researcher who was trained and has had experience in this research field. Thematic analysis was used manually to 
find the common themes. Data of transcription was recorded in text file and coded and identified after reading and re-reading transcriptions by Excel. Similar codes that made same meaning were combined to make sub-themes and similar sub-themes were combined finally to identify common themes (Berkowitz, 1997). The investigator triangulation was used by three researchers looking at the themes. The common themes and sub-themes were confirmed. The consolidated criteria for reporting a qualitative research checklist were used (Tong et al., 2007).

Demographic characteristics and risk of anxiety and depression based on the HADS were described. Since the performance of using HADS to screen mental health problem has not been reported among MDR-TB patients in Thailand, the HADS score was not included in subject selection criteria in this study. The patient's score was not known to the interviewer before the interview to avoid biasing the interviewer. The HADS score was only used as additional information to be considered with the information gained from the in-depth interview. The consistency between self-assessment HADS result and the in-depth interview results was also explored.

\section{Ethics Statement}

The study was approved by the ethics committee of the Faculty of Tropical Medicine, Mahidol University (approval number TMEC 18-015) and by the Ethics Committee of the participating hospital (approval number 119, research number 14/61). The researcher provided an information sheet to all study participants. All participants signed informed consent forms.

\section{Findings}

\section{Participant Characteristics}

A total of 20 MDR-TB patients were recruited for the indepth interviews; seven of them were in the intensive treatment phase. The participants had duration of treatment ranged from 2 to 36 months, with a mean of 14 months. Eleven participants were female. The participants' ages ranged from 19 to 69 years with a mean of 41.45 years, and more than half $(n=11)$ had a primary school education (up to grade 6 in Thailand). Seven participants were unemployed after knowing diagnosis. Three participants were living in slum areas, such as worker camps. Seven participants were living in extended families. Nine participants reported that they did not have any financial support during the treatment.

The HADS score screening test suggested that five participants had anxiety and depression. Eight participants were doubtful cases (HADS score of 8-10). Sixteen participants had a comorbidity, such as diabetes mellitus, hypertension, heart disease, hepatitis, gouty arthritis, anemia, and asthma. Smoking and alcohol drinking were reported in eight and 11 participants, respectively. This study did not attempt to quantify levels of smoking or alcohol intake. All patients received MDR-TB treatment following the national treatment guideline, in which the drug regimen was determined according to drug sensitivity test result, treatment response, and drug tolerability. Adverse drug reactions, including seizure, optic neuritis, psychosis, gastritis, hypothyroidism, arthralgia, nausea and vomiting, peripheral neuropathy, and weight loss, were reported in 16 participants. One participant developed psychosis and had suicidal ideas after taking cycloserine that is known to have psychiatric side effects. Two main themes and seven sub-themes emerged to describe the results: life experiences in confronting MDRTB illness, anxiety, and depression management.

\section{Theme I: Life Experiences in Confronting Multi-Drug-Resistant Tuberculosis}

All participants reported that having MDR-TB was an unpleasant experience. The MDR-TB patients had to confront many difficulties throughout the treatment process. The duration of the coping process usually depends on the individual and their support. According to the indepth interviews, the MDR-TB patients had to confront with the four major difficulties; the emotional storm at the time of diagnosis, insufficient knowledge, negative impact of MDR-TB, and fear of stigmatization.

\section{Emotional Storm at the Time of Diagnosis}

The participants were confused, fearful, and shocked when they first knew about the diagnosis: "I was shocked when first knowing about the diagnosis results, and I felt a lot of stress. I did not want to drop my studies". Another participant cried while she said, "I was confused when the physician told me that I had MDR-TB illness ... I felt chaos in my head and felt like unable to breath." Some participants also reported that knowing the diagnosis result made them feel shock and loneliness in a dark world.

\section{Insufficient Knowledge}

Most participants reported having insufficient knowledge about the MDR-TB illness. They did not know about symptoms and the potential severity of the illness. The only thing they knew was that the disease required longterm treatment for about 2 years: "I did not know about it before, (weep ...) I only knew that it needs both injected and oral drugs for two years". Some participants gained knowledge about the disease from known MDR-TB patients. 
As one patient said, "My aunt had TB before, she was completely cured. I got the disease, and then it shall pass."

\section{Negative Impact of Multi-Drug-Resistant Tuberculosis}

The negative impact of MDR-TB can have potential effects on person's physical, mental, social, and economic status. The participants had negative attitudes toward MDR-TB illness such as fear, hopelessness, sadness, anxiety, and depression. Such negative attitudes increased when learning that the disease was easily transmitted from one person to another. This participant described hopelessness: "I thought I had only a 50\% chance or even less to be cured. Ifeel hopeless and so difficult. The doctor told me that I needed about 6 months of treatment, but 3 years have passed ... nowadays, I'm still not fully recovered. This made me worried that I will die soon." The participant felt terrible, sad, upset, and depressed during the MDR-TB treatment: "For me, the disease is disgusting. I annoy myself and I always think about taking overdose medication to kill myself. But I did not do it because if I did, my life insurance will not pay for a suicidal cause. I did not want to put my sister into troubles."

Treatment for MDR-TB requires both injected and oral medications. These drugs usually cause side effects. Most participants described both physical and mental suffering. The medication often causes nausea and vomiting. The participants could rarely eat and subsequently felt tired and weak. The physical effects were sometimes very serious, especially during the intensive treatment phase and among patients who had underlying diseases, such as diabetes mellitus and hepatitis in particular. MDR-TB medications, particularly cycloserine, have been reported to have serious psychiatric side effects. A few patients reported that they felt hopeless and had thoughts of suicide, which could potentially be due to the medication. "I had no one. I did not want to live. I want to escape from this disease. After taking the pills, I felt unhappy."

Multi-drug-resistant tuberculosis can have potential effects on a patient's life. Some patients could not continue their work or education once they were diagnosed with MDR-TB. They felt loss of their future opportunities: "I passed an entrance exam into an engineering program in a famous university. Though I had a high score, I could not attend the university. MDR-TB ended my future." Another participant confirmed that MDR-TB was the worst thing in her life: "I had MDR-TB during the third year in the university, I had to drop, and I hope that I can go back to school once I am cured." In addition, the participants experienced economic difficulties because they could not work during the first 2 or 3 months after starting the treatments. They lost their jobs and had no income. "I feel very stressful. I'm not rich. If I can 't work, where I can get the money for buying foods and how I survive."

\section{Fear of Stigmatization}

Multi-drug-resistant tuberculosis is highly stigmatized and made the participants feel discouraged. The stigma occurs when people were afraid that the MDR-TB patients will transmit the disease to other people in the community. "People around me who knew that I had MDR-TB, they treated me as I am a disgusted person. When they gazed to me, If felt being stigmatized. " Some participants did not let others know that they had MDR-TB. Stigmatization also led to self-isolation. "People in the community did not know that I have the disease. I did not tell them because they will object me. I did not participate in any community events. I stayed only at home."

\section{Theme 2: Anxiety and Depression Management}

\section{Awareness and Acknowledgment of Stressors}

The HADS screening test suggested that five participants had anxiety and depression. Only one of five patients did not show anxiousness during the interview. This participant said "I am sure that I will get a successful treatment. I protected others by wearing a mask and separated all things from them ... dishes, spoons ... but I still sleep with my wife ... she does not want to separate from me." This patient had suicidal thoughts during the intensive phase, but at the time of the interview the patient felt that the worst situations had already passed. Four participants said that they had suicidal ideas. One participant cried when talking with the interviewer: "Why do people feel disgust when they see me? They do not show interest in me. I have had thoughts about ending myself; I did not want myself to burden anybody else." Patients who had strong family or social supports took about 1-2 months for coping or appropriately managing the stress, anxiety, or depression. The shortest coping period was 1 week, whereas the longest coping time was $>1$ year. "I think I can pass bad situation because I have many good things around me, my family encourage me to fight with the disease."

\section{Coping Mechanisms}

The participants dealt with anxiety and depression by maintaining a positive attitude that the disease can be cured. Participants tried to think positively by knowing that their condition is better than others. Most of the participants self-managed by doing favorite activities, such as playing games, watching TV, listening to music, playing on the internet and playing cards, practicing 
meditation, and making merit: "I like to watch video clips, which encourage and power me. I'm sure that I will survive from the disease." They tried to do many things that made themselves not preoccupied and did not leave space for negative thoughts. In addition, they had activities that yielded good physical health as a consequence, such as exercising on a daily basis, eating balanced meals, quitting smoking, and not drinking alcohol. During the intensive phase, they could not eat any food, but they tried to eat some fruits and take vitamins instead. One participant finally found a solution to help her take the medication more easily by taking the pills with sour tamarind. Participants tried to exercise daily if they were not fatigued. Most of the participants liked to talk with a loved and trusted person that made them feel relaxed. Moreover, religious practices were used for stress management, such as praying, listening to Dhamma talks, and making merits by going to temples and preparing foods for monks. Peer support group was not available in the area, and only one patient consulted a psychologist for proper treatment.

\section{Influence of Family and Social Support}

Strong family support encouraged the member with MDR-TB to cope with the disease, especially during the first 2-3 months of starting the treatment when the severity of side effects of treatment was typically the worst: "Encouragements from my family were the most powerful support for me. I had TB before and was then cured. Then, I developed MDR-TB. My family helped me to face the disease again. At first, I felt so much fear. Now, I am not afraid." One participant confirmed that their family was a key to successfully coping with MDR-TB. "My family's encouragement helped me to get through this worst time in my life." Some participants received financial support from their family when they could not work and did not have any income.

On the other hand, participants who had poor family support tended to inappropriately manage their anxiety. Four participants reported that they had suicidal ideas; all of them had poor family support. The family members were afraid of disease transmission and did not allow the participants to contact with them. "My sister did not permit me to use her toilet after I was diagnosed with $M D R-T B$." In addition, participants did not get a good care or encouragement from spouse or other family members, which made them feel lonely and depressed. "My husband did not support me, he was alcoholic. He was not responsible for the family."

\section{Discussion}

Mental health problems are common among persons with chronic diseases that require long-term treatment (Aydin \& Ulusahin, 2001). Despite this established association, particularly with the stigmatized disease, this problem is often still neglected. People living with MDR-TB require long-term treatment, and they also suffer from the side effects of medications. The results of this qualitative study suggested that persons with MDR-TB had a difficult life experience in coping with the disease throughout their treatment period. Unfortunately, this mental health problem has not received sufficient attention in MDR-TB case management.

Most of the participants in this study had a low educational level, and some were unemployed. Moreover, a majority of the participants had comorbid diseases, such as diabetes and HIV infection. These characteristics are typically observed among MDR-TB patients and may lead to poor MDR-TB treatment outcomes (Jitmuang et al., 2015; Podewils et al., 2011).

An adverse emotional response usually occurs when these patients first learn of their diagnosis. Poor knowledge about the disease can intensify bad feelings, such as sadness, anxiety, fear, shock, and depression (Zhang et al., 2014). A previous study reported that sadness and loneliness may lead to suicidal attempt (Kim, 2014). In the present study, most of the participants who had strong negative feelings had little to no knowledge about MDR-TB. The emotional storm following their diagnosis was often worsened when they learned about the long-term treatment plan that requires both injected and oral medications.

In TB-endemic countries, health facilities have to provide health services to a number of patients each day. Therefore, health personnel may not have sufficient time to thoroughly explain the disease to the MDR-TB patients (Furin et al., 2020). Access to psychiatrists or other mental health providers is still limited, especially in provincial areas (Udomratn, 2007). Other members of the health care team, such as social workers, can play an important role in providing pre-diagnosis or pre-treatment counseling to reduce patient's emotional difficulties when knowing the diagnosis (Furin et al., 2020; Zelnick et al., 2018). Human resources in mental healthcare should be strengthened at the MDR-TB clinics.

Fear of stigmatization is a common emotional response in persons living with MDR-TB, particularly since the disease is contagious (Watkins \& Plant, 2004). Our results showed that stigmatization could lead to patients isolating themselves from family members and communities, leading to severe depression. A previous systematic review also reported that the perception by others that an individual is contagious is a leading cause of stigmatization. Several interventions have been suggested to reduce TB stigma (Courtwright \& Turner, 2010). Peer support among patients has been shown to effectively empower patients to withstand critical judgment internally and externally (Snyman et al., 2018). Moreover, health care providers should educate about disease transmission 
and prevention strategies in communities, which might help decrease the community stigmatization of affected individuals.

The patients with MDR-TB in our study experienced other difficulties in their lives. Some patients could not continue or pursue higher education. Severe symptoms can result in physical disabilities and subsequent loss of employment. A previous study reported that $38 \%-92 \%$ of people living with MDR-TB lost income (van den Hof et al., 2016). This economic impact could potentially add up and affect a patient's mental health (Morris et al., 2013). Although the government provides free treatment services for all MDR-TB patients, financial aid for living expenses should also be considered for those who face economic loss due to illness.

Treatment of MDR-TB also has an effect on patients' mental health directly and indirectly. Medications for MDR-TB treatment, such as cycloserine, have been reported to have psychological side effects ranging from mild depression to psychosis (Torun et al., 2005). In the present study, one patient who received cycloserine had suicidal ideation, so the drug treatment regimen was changed in this patient. In addition, patients may suffer from the long-term MDR-TB treatment regimen that includes injection drugs. Adverse drug reactions associated with MDR-TB treatment can cause fatigue, tiredness, and physical disability, which could lead to anxiety and depression (Thomas et al., 2016). Four patients in this study said that they had suicidal ideation specifically related to the suffering from MDR-TB illness and side effects of treatment. Therefore, patients who received medication with known psychological side effects or those with severe side effects should be closely monitored for their mental health.

According to the theory of psychological stress and coping by Lazarus and Folkman, two coping strategies that persons often use to deal with stress are emotional focus and problem-focus coping (Biggs et al., 2017). In this study, the persons living with MDR-TB took about $1-$ 2 months to cope with their new situation. The emotionfocus strategy can involve such things as trying to accept the disease, seeking support from family and friends, going to religious places, and engaging in hobbies and are often used by patients to cope with the emotional difficulties when first learning of their diagnosis. Religious beliefs in Thai about "Karma" have often been used from the Buddhist tradition to explain the difficulties of life and help people to accept their destiny (Xu, 2018). In this study, religious activities such as praying, listening to Dhamma talks, and making merits by going to temples were commonly used by the participants to help coping with the emotional difficulties. This approach is supported by the theory that emotional focus is likely used by individuals to cope with situations that are unchangeable (McLeod, 2015; Naeem et al., 2012). The problem-focus strategy was commonly used to cope with stress and suffering from MDR-TB treatment. The patients tried to find supplementary foods, vitamins, and fruit that helped them gain more strength. Some patients changed their lifestyle to be healthier and followed their doctor's advice. Unfortunately, individual counseling and peer support groups are not popular in Thailand. Persons living with MDR-TB tend to seek advice from persons they trust such as family members and close friends. Understanding the methods that patients usually used to manage their stress would be useful for planning effective programs to assist patients to cope with their psychological stress.

The findings of this study also showed that family and social support was a critical factor that helped the persons living with MDR-TB cope with stress. The initial coping periods were shorter for patients with strong family support, mentally and financially than for patients without such strong support. The patients who reported having suicidal ideation were discriminated against and isolated from their families and communities. A previous study reported that support from family members, friends, health care workers, and even from other MDR-TB patients could potentially empower the patients to cope with difficult situations during the treatment (Muralidharan et al., 2021; Snyman et al., 2018). To reduce the emotional difficulties associated with learning of their diagnosis, patients and family should be given sufficient information about the disease regarding its contagiousness and recommended treatment plan. For patients with poor family support, establishment of a peer support group would be useful. In this study, the HADS screening tool identified five potential persons with anxiety and depression. The HADS results corresponded with the information gathered from the interview; four out of five definite cases had suicidal ideas during the course of treatment. The Thai version of HADS has been developed and tested (Nilchaikovit et al., 1996). The assessment has been commonly used in chronic non-communicable diseases (Hanprasertpong et al., 2017; Limampai et al., 2017; Sittivarakul \& Wongkot, 2019). Our findings showed that the HADS assessment could be a useful tool to initially screen and monitor mental health of MDR-TB patients. Early detection and treatment of mental health illnesses would potentially improve the MDR-TB treatment outcome as well as the quality of life of the patients.

\section{Limitations of the Research}

This study was conducted in a hospital in Thailand with the highest prevalence of MDR-TB in the country. While the purpose of qualitative research is not to obtain a sample, whose findings are generalizable to the target population under study. We did aim to use a purposive quota sampling method that would allow us to examine 
the life experiences of patients with diverse characteristics, and perhaps extend those findings to others who also have MDR-TB. However, our sample was recruited from one major tertiary hospital in an urban setting where the results may not be as relevant to those living in rural areas or whose access to healthcare is limited.

Potential response bias may have occurred with the HADS assessment, where some people may not be as willing to fully disclose their emotional state. Illiterate persons who had someone read the questions for them this could have had their responses influenced by the interview process. Future quantitative studies at a larger scale are suggested to quantify the burden of mental health problems among persons living with MDR-TB and to confirm the usefulness of HADS assessment among this population.

\section{Conclusion}

The patients with MDR-TB had to face many difficulties throughout their treatment period, which often had an adverse effect on their mental health and treatment outcomes. Therefore, MDR-TB patients should be screened and closely monitored for mental health problems. The MDR-TB patients may require multiple supports to help them cope with the difficulties. Family and peer support are an important key factor associated with successful management of stress in these patients. The proper health education related to the illness and treatment plan should be provided to the patients along with their family members. There is a need to improve access to counseling and mental healthcare among Thai people living with MDR-TB. In addition, the screening and management of mental health disorders should be included in the national MDR-TB case management guidelines to provide better quality of care in the country.

\section{Acknowledgments}

The authors thank the MDR-TB patients and health care providers at the TB clinic in the participating hospital, Thailand. We would like to thank an English editor from the Enago ${ }^{\mathrm{TM}}$ editing service group. We are also grateful to Mr. Irwin F. Chavez, Department of Tropical Hygiene, Faculty of Tropical Medicine, Mahidol University for his support on an additional English editing of the manuscript.

\section{Declarations of Conflicting Interests}

The author(s) declared no potential conflicts of interest with respect to the research, authorship, and/or publication of this article.

\section{Funding}

The author(s) disclosed receipt of the following financial support for the research, authorship, and/or publication of this article:
This study was funded by the Young Researcher Development Program from the National Research Council of Thailand and the Faculty of Tropical Medicine, Mahidol University, Thailand.

\section{Author's Note}

This was not a mixed-methods study. The HADS score was used to describe participants as a part of the demographic data.

\section{ORCID iDs}

Samorn Numpong (D) https://orcid.org/0000-0003-2684-7958

Saranath Lawpoolsri (D) https://orcid.org/0000-0003-4841-7924

\section{References}

Alene, K. A., Clements, A. C. A, McBryde, E. S., Jaramillo, E., Lönnroth, K., \& Shaweno, D. (2018). Mental health disorders, social stressors, and health-related quality of life in patients with multidrug-resistant tuberculosis: A systematic review and meta-analysis. Journal of Infection, 77(5), 357-367. http://doi.org/10.1016/j.jinf.2018.07.007

Aydin, I. O., \& Ulusahin, A. (2001). Depression, anxiety comorbidity, and disability in tuberculosis and chronic obstructive pulmonary disease patients: Applicability of GHQ-12. General Hospital Psychiatry, 23(2), 77-83. http://doi.org/10.1016/s0163-8343(01)00116-5

Berkowitz, S. (1997). User-friendly handbook for mixed method evaluations: Part II. overview of qualitative methods and analytic techniques: Chapter 4: Analyzing qualitative data. https://www.nsf.gov/pubs/1997/nsf97153/chap_4.htm

Biggs, A., Brough, P., \& Drummond, S. (2017, February 18). Lazarus and Folkman's psychological stress and coping theory. https://onlinelibrary.wiley.com/doi/abs/10.1002/ 9781118993811.ch21.

Borisov, S., Danila, E., Maryandyshev, A., Dalcolmo, M., Miliauskas, S., Kuksa, L., Manga, S., Skrahina, A., Diktanas, S., Codecasa, L. R., Aleksa, A., Bruchfeld, J., Koleva, A., Piubello, A., Udwadia, Z. F., Akkerman, O. W., Belilovski, E., Bernal, E., Boeree, M. J., \& Migliori, G. B. (2019). Surveillance of adverse events in the treatment of drug-resistant tuberculosis: First global report. European Respiratory Journal, 54(6), 1901522. http://doi.org/10. $1183 / 13993003.01522-2019$

Bureau of Tuberculosis. (2015). Guideline for programmatic management of drug-resistant tuberculosis. The Agricultural Cooperative Federation of Thailand. Limited.

Courtwright, A., \& Turner, A.N. (2010). Tuberculosis and stigmatization: Pathways and interventions. Public Health Reports, 125(4), 34-42. https://doi.org/10.1177\% 2F00333549101250S407

Furin, J., Loveday, M., Hlangu, S., Dickson-Hall, L., le Roux, S., Nicol, M., \& Cox, H. (2020). "A very humiliating illness": A qualitative study of patient-centered care for RifampicinResistant Tuberculosis in South Africa. BMC Public Health, 20(1), 76. https://doi.org/10.1186/s12889-019-8035-z 
Grenard, J. L., Munjas, B. A., Adams, J. L., Suttorp, M., Maglione, M., McGlynn, E.A., \& Gellad, W. F. (2011). Depression and medication adherence in the treatment of chronic diseases in the United States: A meta- analysis. Journal General Internal Medicine, 26(10), 1175-1182. http://doi.org/10.1007/s11606-011-1704-y

Hanprasertpong, J., Geater, A., Jiamset, I., Padungkul, L., Hirunkajonpan, P., \& Songhong, N. (2017). Fear of cancer recurrence and its predictors among cervical cancer survivors. Journal of Gynecologic Oncology, 28(6), e72. http://doi.org/0.3802/jgo.2017.28.e72

Jiraphongsa, C., Wangteeraprasert, T., Henpraserttae, N., Sanguanwongse, N., Panya, L., Sukkasitvanichkul, J., \& Pittayawonganon, C. (2011). Community outbreak of multidrug resistance tuberculosis Kanchanaburi Province, Thailand on 2002-June 2010. Journal of Preventive Medicine Association of Thailand, 1(3), 261-271.

Jitmuang, A., Munjit, P., \& Foongladda, S. (2015). Prevalence and factors associated with multidrug-resistant tuberculosis at Siriraj Hospital, Bangkok. Thailand. The Southeast Asian Journal Tropical Medicine and Public Health, 46(4), 697-706.

Kim, Y. (2014). Understanding the life experiences of older adults in Korea following a suicide attempt. Qualitative Health Research, 24(10), 1391-1399. https://doi.org/10. 1177\%2F1049732314547643

Kittyanun, C. (2007). Antituberculosis drug resistance in Makarak Hospital. Region 6-7 Medical Journal, 26(4), 351-356.

Lambert, V. A., \& Lambert, C. E. (2012). Qualitative descriptive research: An acceptable design. Pacific Rim International Journal of Nursing Research, 16(4), 255-256. https://he02. tci-thaijo.org/index.php/PRIJNR/article/view/5805

Limampai, P., Wongsrithep, W., \& Kuptniratsaikul, V. (2017). Depression after stroke at 12- month follow-up: A multicenter study. International Journal of Neuroscience, 127(10), 887-892. http://doi.org/10.1080/00207454.2016. 1277344

McLeod, S. (2015). Stress Management. Simply Psychology. https://www.simplypsychology.org/stress-management. html\#em

Morris, M. D., Quezada, L., Bhat, P., Moser, K., Smith, J., Perez, H., Laniado-Laborin, R., Estrada-Guzman, J., \& Rodwell, T. C. (2013). Social, economic, and psychological impacts of MDR-TB treatment in Tijuana, Mexico: A patient's perspective. The International Journal of Tuberculosis and Lung Disease. 17(7), 954-960. http://dx.doi.org/10.5588/ ijtld.12.0480

Muralidharan, A., Peeples, A., \& Lucksted, A. (2021). Health behavior change processes among adults with serious mental illness engaged in illness self-management. Qualitative Health Research, 31(6), 1155-1168. http://doi.org/ 10.1177/1049732321992049

Naeem, F., Ayub, M., Kingdon, D., \& Gobbi, M. (2012). Views of depressed patients in Pakistan concerning their illness, its causes, and treatments. Qualitative Health Research, 22(8), 1083-1093. https://doi.org/10.1177\%2F1049732312450212

Nilchaikovit, T., Lortrakul, M., \& Phisansuthideth, U. (1996). Development of Thai version of the hospital anxiety and depression scale in cancer patients. Journal of the Psychiatris Association Thailand, 41(1), 18-30.

Podewils, L. J., Holtz, T., Riekstina, V., Skripconoka, V., Zarovska, E., Kirvelaite, G., Kreigere, E., \& Leimane, V. (2011). Impact of malnutrition on clinical presentation, clinical course, and mortality in MDR-TB patients. Epidemiology and Infection, 139(1), 113-120. https://doi.org/ 10.1017/S0950268810000907

Sittivarakul, W., \& Wongkot, P. (2019). Anxiety and depression among patients with uveitis and ocular inflammatory disease at a tertiary center in southern Thailand: Vision- related quality of life, sociodemographics, and clinical characteristics associated. Ocular Immunology and Inflamation, 27(5), 731-742. http://doi.org/10.1080/09273948.2018.1484495

Snyman, L., Venables, E., Trivino Duran, L., Mohr, E., Azevedo, V. D., Harmans, X., \& Isaakidis, P. (2018). 'I didn't know so many people cared about me': Support for patients who interrupt drug-resistant TB treatment. The International Journal of Tuberculosis and Lung Disease, 22(9), 1023-1030. http://dx.doi.org/10.5588/ijtld.17.0826

Thanprasertsuk, S., Utaipiboon, C., Tossapornpong, K., Sukhasitwanichkul, J., \& Jullawet, W. (2017). Descriptive epidemiology of multidrug-resistance tuberculosis. Makarak Hospital 2007-2015. Disease Control Journal, 43(4), 400-412. https://doi.org/10.14456/dcj.2017.6.

Thomas, B. E., Shanmugam, P., Malaisamy, M., Ovung, S., Suresh, C., Subbaraman, R., Adinarayanan, S., \& Nagarajan, K. (2016). Psycho-socio-economic issues challenging multidrug resistant tuberculosis patients: A systematic review. PLOS One, 11(1), e0147397. http://doi.org/10.1371/ journal.pone.0147397

Tong, A., Sainsbury, P., \& Craig, J. (2007). Consolidated criteria for reporting qualitative research (COREQ): A 32-item checklist for interviews and focus groups. International Journal for Quality Health in Care,19(6), 349-357. https:// doi.org/10.1093/intqhe/mzm042

Torun, T., Gungor, G., Ozmen, I., Bolukbasi, Y., Maden, E., Bicakci, B., Atac, G., Sevim, T., \& Tahaoglu, K. (2005). Side effects associated with the treatment of multidrugresistant tuberculosis. The International Journal of Tuberculosis and Lung Disease, 9(12), 1373-1377.

Udomratn, P. (2007). Mental health and psychiatry in Thailand. International Psychiatry: Bulletin of the Board of International Affairs of the Royal College of Psychiatrists, 4(1), $11-14$.

van den Hof, S., Collins, D., Hafidz, F., Beyene, D., Tursynbayeva, A., \& Tiemersma, E. (2016). The socioeconomic impact of multi-drug resistant tuberculosis on patients: Results from Ethiopia, Indonesia and Kazakhstan. BMC Infectious Disease, 16(1), 470. https://doi.org/10.1186/s12879-016-1802-х 
Vega, P., Sweetland, A., Acha, J., Castillo, H., Guerra, D., Smith Fawzi, M. C., \& Shin, S. (2004). Psychiatric issues in the management of patients with multidrug-resistant tuberculosis. The International Journal of Tuberculosis and Lung Disease, 8(6), 749-759.

Walker, IF., Khanal, S., Hicks, JP., Lamichhane, B., Thapa, A., Elsey, H., Baral, S. C., \& Newell, J. N. (2018). Implementation of a psychosocial support package for people receiving treatment for multidrug-resistant tuberculosis in Nepal: A feasibility and acceptability study. PLOS One, 13(7), 1-15. https://doi.org/10.1371/journal.pone.0201163

Watkins, R. E., \& Plant, A. J. (2004). Pathways to treatment for tuberculosis in Bali: Patient perspectives. Qualitative Health Research 14(5), 691-703. http://doi.org/10.1177/ 1049732304263628

World Health Organization. (2014). Companion handbook to the WHO guidelines for the programmatic management of drug-resistant tuberculosis. www.who.int
Xu, J. (2018). Buddhism-as-a-meaning-system for coping with late-life stress: A conceptual framework. Aging \& Mental Health, 22(1), 100-108. http://doi.org/10.1080/13607863. 2016.1227767

Zelnick, J. R., Seepamore, B., Daftary, A., Amico, K. R., Bhengu, X., Friedland, G., Padayatchi, N., Naidoo, K., \& O'Donnell, M. R. (2018). Training social workers to enhance patient-centered care for drug-resistant TB-HIV in South Africa. Public Health Action, 8(1), 25-27. http://doi. org/10.5588/pha.17.0114

Zhang, Q., Liao, J., Liao, X., Wu, X., Wan, M., Wang, C., \& Ma, Q. (2014). Disease knowledge level is a noteworthy risk factor of anxiety and depression in patients with chronic obstructive pulmonary disease: A cross-sectional study. BMC Pulmonary Medicine, 14(1), 92. http://doi.org/10.1186/1471-2466-14-92

Zigmond, A. S., \& Snaith, R. P. (1983). The hospital anxiety and depression scale. Acta Psychiatrica Scandinavica, 67(6), 361-363. http://doi.org/10.1111/j.1600-0447.1983.tb09716.x 